\title{
Efectos de un programa de juegos reducidos en la percepción subjetiva del esfuerzo en una muestra de chicas adolescentes
}

\author{
Effects of a small sided games program on rate of \\ perceived exertion in a sample of teenage girls
}

\section{Efeitos de um programa de jogos reduziu no esforço percebido em uma amostra de adolescentes}

\author{
Inmaculada Martín Martínez ${ }^{1}$, Rafael Enrique Reigal Garrido ${ }^{2}$, Luis Javier Chirosa Ríos ${ }^{1,2}$, Antonio Hernández Mendo ${ }^{3}$, \\ Ignacio Chirosa Ríos ${ }^{1,2}$, Ignacio Martín Tamayo ${ }^{1,2}$ y Rafael Guisado Barrilao ${ }^{4}$
${ }^{1}$ Facultad de Ciencias de la Actividad Física y el Deporte. Universidad de Granada, ${ }^{2}$ Grupo de Investigación CTS-642. Universidad de Granada, ${ }^{3}$ Facultad de Psicología. Universidad de Málaga y ${ }^{4}$ Facultad de Ciencias de la Salud. Universidad de Granada.

Resumen: El objetivo de este trabajo fue analizar los efectos de un programa de juegos reducidos, con una duración de ocho semanas, sobre la percepción subjetiva del esfuerzo. La muestra estuvo compuesta por 42 chicas adolescentes del municipio de Priego de Córboba (Córdoba, España), con edades entre los 15 y 16 ańos y físicamente inactivas. Se utilizó un diseńo cuasi-experimental con dos grupos aleatorizados, control y experimental. Los instrumentos utilizados fueron la Escala de Percepción Subjetiva de Esfuerzo de Borg, pulsómetros y el Game Perfomance Assessment Instrument (GPAI). Los resultados indicaron un descenso significativo en la percepción de esfuerzo e incrementos en la participación y eficacia del juego, sin existir diferencias en las frecuencias cardiacas de trabajo. Estos resultados sugieren que el aprendizaje y adaptación a los procesos de toma de decisión podrían estar en la base de este fenómeno.

Palabras clave: actividad física; adolescencia; percepción subjetiva del esfuerzo; juegos reducidos.

Resumen: The aim of this study was to analyse the effects of a small sided games program, over a period of eight weeks, on rate of perceived exertion. The participants in the study were 42 girls from Priego of Córdoba (Córdoba, Spain), between 15 and 16 years of age, and physically inactive. The research was based on a quasi-experimental design with two randomised groups, control and experimental. The instruments used to evaluate were the Borg Rate of Perceived Exertion Scale, heart rate monitors and the Game Perfomance Assessment Instrument (GPAI). Results showed a significant decrease in perceived exertion and increases in participation and effectiveness in the game, but without differences in working heart rates. These results suggest that learning and adapting to the decision-making process could be the cause of these events.

Palabras clave: actividad física; adolescencia; percepción subjetiva del esfuerzo; juegos reducidos.

Resumo: O objetivo deste estudo foi analisar os efeitos de um programa de jogos reduzidos, com duração de oito semanas, sobre a percepçáo subjetiva do esforço. A amostra foi composta por 42 adolescentes do sexo feminino no município de Priego de Córboba (Córdoba, Espanha), com idade entre 15 e 16 anos de idade e fisicamente inativos. Foi utilizado um desenho quasi-experimental com dois grupos aleatórios, controle e experimental. Os instrumentos utilizados foram a Escala de Percepção subjetiva do Esforço de Borg, monitores de freqüência cardíaca e Game Perfomance Assessment Instrument (GPAI). Os resultados indicaram uma diminuiçáo significativa na percepção de esforço e aumentos na participação e eficácia do jogo, não há diferenças nas freqüências cardíacas. Estes resultados sugerem que a aprendizagem e adaptação aos processos de tomada de decisão poderia estar na raiz desse fenômeno.

Palavras-chave: atividade física; adolescência; percepçẫo subjetiva do esforço; jogos reduzidos.

\section{Introducción}

El control de la carga de trabajo durante un ejercicio físico puede ser evaluado a través de diferentes herramientas La magnitud de la carga se mide a través de índices internos y externos. Entre los índices de naturaleza interna, se pueden citar el consumo de oxígeno $\left(\mathrm{VO}_{2}\right)$, la frecuencia cardiaca o la concentración de ácido láctico, existiendo otros tantos externos como el tiempo de práctica, la distancia recorrida o

Dirección para correspondencia [Correspondence address]: Inmaculada Martín Martínez. Universidad de Granada. Facultad de Ciencias de la Actividad Física y el Deporte. Carretera de Alfacar s/n, 18071, Granada (Espańa). E-mail: inmartintinez@hotmail.com la velocidad de desplazamiento (Cuadrado-Reyes, ChirosaRíos, Chirosa-Ríos, Martin-Tamayo y Aguilar-Martínez, 2012). La percepción subjetiva del esfuerzo (PSE) (Borg, $1973,1982)$ es una variable psicofísica de control interno que contribuye excepcionalmente a este análisis, siendo sensible a las diferentes demandas de la tarea realizada, así como a la fatiga acumulada durante el ejercicio (Monteiro, Farinatti, Oliveira y Araújo, 2011). De este modo, el uso de esta herramienta se ha extendido en las últimas décadas y ha sido ampliamente utilizada en el ámbito deportivo, tanto individual como colectivo (Faulkner, Parfitt y Eston, 2008; 
Hill-Haas, Coutts, Dawson y Rowsell, 2010; Milanez et al., 2010).

La PSE permite obtener datos sobre cómo se siente una persona cuando realiza un ejercicio físico, pudiéndose contrastar con otros análisis más objetivos, enriqueciendo, así, la evaluación que se efectúe sobre la situación (Borg, 1982). Aunque no tiene el grado de precisión de otras pruebas, se han encontrado relaciones significativas con factores de tipo fisiológico, que indica ser una medida válida y fiable para analizar la intensidad de la actividad y el grado de fatiga (Kilpatrick, Bortzfield y Giblin, 2012; Skatrud-Mickelson, Benson, Hannon y Askew, 2011). Además, tiene la ventaja de ser un método de análisis no invasivo y de fácil acceso, lo que reduce los costes de su administración e incrementa las posibilidades de aplicarlo en diferentes contextos (Alexiou y Coutts, 2008; Casamichana, Castellano, Blanco-Villaseñor y Usabiaga, 2012; Cuadrado-Reyes et al., 2012).

En los deportes colectivos, la PSE puede ser un instrumento muy potente para explicar aspectos relacionados con la adaptación en el rendimiento y permitir ajustar diferentes elementos que afecta a la carga de entrenamiento. De hecho, aunque recoge sensaciones propias del trabajo físico, también integra otras de tipo motivacional, de personalidad o relacionadas con el esfuerzo llevado a cabo para resolver los diferentes problemas tácticos desarrollados durante un ejercicio (Beniscelli y Torregrosa, 2010; Guijarro, de la Vega y del Valle., 2009; Tenenbaum y Hutchinson, 2007). Entre los diferentes factores citados, la toma de decisión es un elemento esencial cuando se quiere analizar la PSE. Estos procesos complejos requieren un intenso y continuo esfuerzo cognitivo para atender las demandas técnico-tácticas de las tareas, así como elaborar la respuesta más adecuada en un contexto de alta variabilidad e incertidumbre continua (Araújo, 2013; Tenenbaum, Basevitch, Gershgoren y Filho, 2013; Vila-Maldonado, García y Contreras, 2012; Vilar, Araújo, Davids y Button, 2012).

En el conjunto de este tipo de deportes, los juegos reducidos (small sided games) son ejercicios que se emplean habitualmente como método de trabajo dirigido dentro de la táctica grupal y complementario debido a su alta versatilidad, estando su uso muy extendido en la actualidad (Casamichana, Castellano y Dellal, 2013; Da Silva et al., 2011; Duarte, Batalha, Folgado y Sampaio, 2009; Hill-Hass et al., 2010; Impellizzeri, Rampinini, Coutts, Sassi y Marcora, 2004). En ellos, se modifican algunos elementos del juego real como el tiempo disponible, el número de jugadores, el espacio útil o las reglas de funcionamiento. Sin embargo, a pesar de dichas alteraciones, se consigue mantener la lógica interna del juego, lo que permite alcanzar interesantes objetivos tanto de tipo físico como técnico-táctico durante los entrenamientos (Abrantes, Nunes, Maças, Leite y Sampaio, 2012; Frencken, Van Der Plaats, Visscher y Lemmink, 2013; Hill-Hass, Dawson, Impellizzeri y Coutts, 2011).
La PSE ha sido utilizada en este tipo de ejercicios para evaluar la intensidad de la carga de entrenamiento, siendo un indicador sensible a las características e implicaciones de la actividad (Coutts, Rampinini, Marcora, Castagna e Impellizzeri, 2009; Cuadrado-Reyes et al. 2012; Hill-Hass et al., 2010; Hill-Hass, Rowsell, Dawson y Coutts, 2009). En este sentido, existen estudios que encontraron cambios en la PSE al modificarse el número de jugadores (Casamichana et al., 2012; Sampaio, Abrantes y Leite, 2009), el área de juego (Casamichana y Castellano, 2010; Köklü, Albayrak, Keysan, Alemdaroğlu y Dellal, 2013; Rampinini et al., 2007), las reglas de juego (Dellal et al., 2012) o la experiencia acumulada en él (Casamichana, San Román-Quintana, CallejaGonzález y Castellano, 2013; Dellal, Hill-Haas, Lago-Penas y Chamari, 2011).

Paralelamente a la percepción del esfuerzo realizado, otras variables como la frecuencia cardiaca han sido utilizadas igualmente para el control del ejercicio en este contexto, observándose cambios en ella tras modificar aspectos como el número de jugadores (Abrantes et al., 2012; Dellal et al., 2008) o las dimensiones del terreno de juego (Casamichana y Castellano, 2010; Toh, Guelfi, Wong y Fournier, 2011). En general, ambas variables han manifestado comportamientos similares en los estudios analizados, aunque en algunos se han apreciado ciertas particularidades (Dellal, Drust y LagoPenas, 2012; Hill-Hass et al., 2011; Rampinini et al., 2007). Como ejemplo, Dellal, Hill-Haas et al. (2011) evaluaron a jugadores de fútbol y observaron que la PSE aumentaba cuando se limitaba el número de toques en un juego 3 vs. 3 y aumentaba la participación en el juego, aunque el incremento de frecuencia cardiaca no fue tan evidente. En otra investigación, efectuada por Dellal, Lago-Penas, Wong y Chamari (2011) se pudo comprobar en un juego de fútbol 4 vs. 4 , que limitar el ejercicio a dos toques, también generaba un aumento de la PSE sin que lo hiciera proporcionalmente la frecuencia cardiaca.

Las conductas desarrolladas durante los juegos reducidos han sido analizadas en diferentes estudios. En ellos, se ha observado que el número de acciones aumentaba si se disminuía el área de juego (Casamichana y Castellano, 2009) o si el número de jugadores era menor (Frencken et al., 2013; Jones y Drust, 2007). Para evaluar la participación y rendimiento de las acciones efectuadas en un deporte colectivo, Oslin, Mitchell y Griffin (1998) diseńaron un instrumento denominado Game Perfomance Assessment Instrument (GPAI). En concreto, diversos estudios han utilizado el GPAI en contexto escolar después de periodos de enseñanza en los que se utilizaron los juegos reducidos dentro del entrenamiento de fútbol (Harvey, Cushion, Wegis y Massa-Gonzalez, 2010; Mesquita, Farias y Hastie, 2012), fútbol-sala (Corrêa, Da Silva y Paroli, 2004) o balonmano (Balakrishnan, Rengasamy y Aman, 2011).

No existen demasiados estudios en los que se examine de 
manera sistemática el impacto de la toma de decisiones en la PSE, aunque sí se pueden encontrar algunas investigaciones que han indagado en la relación entre estas variables en los juegos reducidos (Abrantes et al., 2012; Dellal, Chamari et al., 2011; Hill-Hass et al., 2011). Como ejemplo, Dellal, HillHaas et al. (2011) realizaron un estudio con jugadores de fútbol en el que observaron, tanto en juegos reducidos 2 vs. 2, 3 vs. 3 y 4 vs. 4, que cuando se limitaba el número de contactos con el balón existía un mayor número de acciones durante el juego y aumentaba la PSE. De igual forma, Dellal et al. (2012) efectuaron otro estudio con jugadores de fútbol en el que observaron valores más altos en la percepción de esfuerzo y en la intensidad del ejercicio cuando participaban en juegos reducidos 4 vs. 4 con un número libre de toques respecto a la obligatoriedad de golpear sólo una o dos veces el balón.

Hasta la fecha, la literatura existente no ha resuelto completamente, en el contexto de los juegos reducidos, la cuestión sobre el impacto que tiene la toma de decisión en la PSE. Por ello, el presente estudio pretende analizar los cambios en la PSE en un programa de juegos reducidos 3 vs. 3, evaluando como medidas de control la frecuencia cardiaca, así como la participación en el juego y la eficacia en la toma de decisiones. Así, el objetivo principal de este trabajo fue determinar si la adaptación a las diferentes tareas que el jugador debe resolver durante la práctica deportiva tuvo efectos sobre la percepción del esfuerzo realizada.

\section{Método}

\section{Participantes}

Participaron en la investigación 42 mujeres adolescentes del municipio de Priego de Córdoba (Córdoba, España), con edades comprendidas entre los 15 y 16 ańos $(\mathrm{M} \pm \mathrm{DT}$ : edad= $15.36 \pm .48$ ańos; altura $=161.10 \pm 5.49 \mathrm{~cm}$; peso $=60.85 \pm$ $10.84 \mathrm{~kg} ; \mathrm{IMC}=23.43 \pm 4.02 \mathrm{~kg} \cdot \mathrm{m}^{-2}$; masa grasa $=27.58 \pm$ $8.36 \%$; masa magra: $42.82 \pm 6.12 \%$; FCmax $=196.21 \pm$ $6.42 \mathrm{ppm}$; VO2 $\left.\mathrm{max}=38.93 \pm 3.57 \mathrm{ml} \cdot \mathrm{kg}^{-1} \cdot \mathrm{min}^{-1}\right)$. La muestra se seleccionó de un centro escolar de dicha localidad, donde se efectuaron la intervención y las mediciones pertinentes. No se incluyeron en el estudio aquellos adolescentes que habían repetido curso y los que realizaban actividad física de forma regular más de 2 días a la semana además de las clases de educación física, para evitar que la práctica moderada e intensa de ejercicio físico generase interferencias en los datos. Asimismo, la existencia de patologías que pudieran influir en los resultados se consideró un criterio de exclusión.

\section{Instrumentos}

a) Para evaluar la PSE se utilizó la Escala de Percepción Subjetiva de Esfuerzo, con rango de 6 a 20 (Borg, 1970). Esta escala se utiliza para evaluar el esfuerzo que percibe una persona cuando realiza un ejercicio físico, e integra la información procedente de diferentes sistemas orgánicos, tanto físicos como psicológicos.

b) Para analizar las conductas durante el juego se utilizó el Game Perfomance Assessment Instrument (GPAI) (Oslin et al., 1998), que permite registrar los siguientes aspectos: Toma de decisiones adecuada (TDA) e inadecuada (TDI), ejecución técnica eficaz (ETE) e ineficaz (ETI), apoyo adecuado (AA) e inadecuado (AI), cobertura adecuada (CA) e inadecuada (CI), y marca adecuada (MA) e inadecuada (MI). Posteriormente se transforman en las categorías: Toma de Decisiones (ITD), Ejecución Técnica (IET), Apoyo (IA), Cobertura (IC), Marcaje (IM), Participación en el juego (IPJ) y Rendimiento en el Juego (IRJ). Para este trabajo, se utilizaron únicamente las categorías IPJ e IRJ como medidas de participación y eficacia en el juego. La fiabilidad y validez de este instrumento se ha evaluado de manera positiva en diversos trabajos (Mitchell, Oslin y Griffin, 1995; Wright, McNeill, Fry y Wang, 2005), encontrándose valores en los coeficientes de correlación test-retest entre .84 y .99, así como valores de fiabilidad inter-observadores entre .81 y .83 (Oslin et al. 1998).

c) Se evaluaron tres parámetros de condición física, (1) el consumo máximo de oxígeno $\left(\mathrm{VO}_{2} \mathrm{max}\right)$, a través del test de Course Navette (EUROFIT, 1993; Léger, 1988). Esta prueba consiste en un test incremental de ida y vuelta, sobre una distancia de 20 metros, con un aumento de la velocidad de $0.5 \mathrm{~km}$ cada minuto, partiendo de una velocidad inicial de $8.5 \mathrm{~km} / \mathrm{h}$. Para el cálculo concreto del consumo de oxígeno se aplicó la fórmula VO2max $=31.025+3.238 \mathrm{~V}-3.248 \mathrm{E}+0.1536 \mathrm{VE}$ (siendo $V$ la velocidad alcanzada en la última etapa completada y $E$ la edad del participante); (2) la composición corporal utilizando un bioimpedanciómetro (Tanita ${ }^{\oplus}$ Body Composition Monitor modelo BF-350, Tanita Corporation, Tokyo, Japan); y, (3) la frecuencia cardíaca máxima (FCmáx) y la frecuencia cardiaca (FC) de trabajo durante la actividad, empleando para ello pulsómetros (Polar ${ }^{\ominus}$ modelo S610, Polar Electro Oy, Kempele, Finlandia). Se consideró como FCmáx el valor máximo alcanzado durante el test de esfuerzo y la FC de trabajo el valor medio obtenido durante la participación en los juegos reducidos.

d) Para el registro de imágenes se empleó una cámara SONY ${ }^{\oplus}$ modelo DCR-DVD 505E.

\section{Procedimiento}

Por escrito, se informó a los padres y al centro escolar del estudio que se quería llevar a cabo. Tras ello, se obtuvo el consentimiento informado de los padres y el permiso de la 
dirección del centro. Además, durante el proceso de la investigación se respetaron los principios éticos de la declaración de Helsinki para las investigaciones en seres humanos.

Tras distribuir aleatoriamente la muestra en los grupos control y experimental, se evaluó la composición corporal y consumo de oxígeno máximo. Para obtener los valores de consumo de oxígeno máximo se realizó la prueba de Course Navette por grupos de 10-15 alumnos, empleándose 20 minutos para la evaluación de cada grupo. Por otro lado, para obtener las medidas de composición corporal, se utilizó un bioimpedanciómetro y se evaluó a primera hora de la mańana. Además, con el objetivo de reducir el error, se tuvieron en cuenta unas pautas y recomendaciones como son: no realizar ejercicios agotadores el día anterior, no alterar significativamente la alimentación el día previo a la prueba, vestir ropa cómoda, controlar la toma de medicamentos que puedan alterar los niveles de agua corporal y evitar retener líquidos (George, Fisher y Vehrs, 2007).

Además de estas mediciones iniciales, antes y después del programa de intervención se evaluó las conductas de juego en diferentes juegos reducidos mediante el GPAI, la PSE tras cada periodo de trabajo y la FC de trabajo media durante cada periodo de trabajo. Para evaluar las conductas de juego se analizaron tres juegos reducidos 3 vs. 3 (fútbol sala, baloncesto y balonmano) en una sesión de 30 minutos, con una duración de 6 minutos cada juego y un descanso de 1 minuto entre ellos. Estos ejercicios estuvieron precedidos por un calentamiento de 7 minutos basados en ejercicios de activación del sistema vegetativo, movilidad articular y en un juego de 3 vs. 3 en el que había que mantener la posesión de un balón utilizando, para el pase y recepción, las manos. Estos juegos fueron grabados y visionados en dos ocasiones por un observador con una diferencia de tres meses. Para analizar la calidad del dato obtenido se analizó la fiabilidad intraobservador mediante Kappa de Cohen (1960).

Para utilizar la escala de PSE se efectuó un entrenamiento previo de cuatro sesiones en las clases de Educación Física, complementando el aprendizaje a través de lecturas de las instrucciones de la prueba (Feriche, Chicharro, Vaquero, Perez y Lucia, 1998). Además, el test de Course Navette fue utilizado para completar el entrenamiento de la escala de PSE, aplicándose una quinta sesión de formación durante la misma. Tras cada ejercicio, antes y después del calentamiento, así como cinco minutos después de terminar el último ejercicio, los participantes anotaron en una plantilla elaborada ad hoc los valores de PSE. Estos datos fueron registrados en carpetas individuales para evitar interferencias entre ellos (CuadradoReyes et al. 2012).

El programa de intervención consistió en aumentar la práctica física semanal en 90 minutos, divididos en una sesión de 60 minutos y otra de 30 (implementadas en días diferentes a las clases de Educación Física y en horario escolar), pasando de 120 minutos (clases de educación física ordinarias) a 210 minutos semanales. Durante el programa, ambos grupos siguieron las sesiones programadas en clases de educación física, divididas en un primer bloque de deportes colectivos (enseñanza del baloncesto) y un segundo de expresión corporal (aerobic y bailes modernos). El grupo experimental, en la sesión de complementaria de 60 minutos se efectuaron seis juegos 3 vs. 3 (dos de fútbol-sala sin portero, dos de baloncesto y dos de balonmano sin portero) y en la sesión complementaria de 30 minutos tres juegos 3 vs. 3 (uno de cada modalidad deportiva), con una duración de 6 minutos cada uno y un descanso de 1 minuto entre ellos. Ambas estuvieron precedidas por un calentamiento de 7 minutos basados en ejercicios de activación del sistema vegetativo, movilidad articular y en un juego de 3 vs. 3 en el que había que mantener la posesión de un balón utilizando, para el pase y recepción, las manos. El área de juego total fue de $240 \mathrm{~m}^{2}(20 \times 12 \mathrm{~m})$, correspondiendo $40 \mathrm{~m}^{2}$ a cada jugador. La FC media con la que se participó fue de $175.47 \pm 11.76$ ppm $(80.65 \pm 8.04 \%$ FCR $)$ y la percepción media de esfuerzo de $13.37 \pm 1.56$. Por su parte, el grupo control no realizó ejercicio físico durante este tiempo, dedicándolo a efectuar trabajos escritos y lecturas sobre aspectos relacionados con los contenidos de Educación Física.

\section{Análisis de los datos}

Se realizaron análisis de la varianza (ANOVA) bifactorial para cada medida de PSE, salvo para las previas al calentamiento, y los índices IPJ e IRJ del GPAI, siendo un primer factor el grupo con dos niveles (control y experimental) y un segundo factor la variable pre-post con dos niveles (valores previos y tras la intervención). Se estudiaron los efectos principales y la interacción entre variables. La significación de los efectos se analizó mediante comparaciones de Bonferroni. Para analizar los valores de percepción de esfuerzo previos al calentamiento, dada la falta de distribución normal tras analizar la normalidad con la prueba de Kolmogorov-Smirnov y estudiar tanto la asimetría como la curtosis, se aplicaron las técnicas no paramétricas U de Mann-Whitney y Wilcoxon. Para analizar la fiabilidad intraobservador y evaluar la calidad del dato obtenido de las observaciones (GPAI), se utilizó la Kappa de Cohen (1960). El programa estadístico utilizado fue el SPSS en su versión 20.

\section{Resultados}

\section{Percepción subjetiva de esfuerzo}

En la tabla 1 se muestran las medias y desviaciones típicas de la PSE (Borg 6-20) para ambos grupos, antes y después del programa de intervención. Asimismo, se indican los valores de asimetría, curtosis y prueba de normalidad KolmogorovSmirnov. 
Tabla 1. Medias y desviaciones típicas para los valores de PSE.

\begin{tabular}{|c|c|c|c|c|c|c|c|c|c|c|c|}
\hline & & \multicolumn{5}{|c|}{ Control } & \multicolumn{5}{|c|}{ Experimental } \\
\hline & & $M$ & $D T$ & $A$ & $K$ & $Z$ & $M$ & $D T$ & $A$ & $K$ & $Z$ \\
\hline \multirow[t]{2}{*}{ Pre-Cal } & Pre & 6.09 & .29 & 3.06 & 8.08 & $2.48^{* * *}$ & 6.05 & .21 & 4.68 & 22.00 & $2.52^{* * *}$ \\
\hline & Post & 6.14 & .35 & 2.27 & 3.49 & $2.41^{* * *}$ & 6.09 & .29 & 3.06 & 8.09 & $2.49^{* * *}$ \\
\hline \multirow[t]{2}{*}{ Post-Cal } & Pre & 10.32 & 1.09 & -.71 & .25 & 1.10 & 10.27 & 1.16 & -.58 & -.54 & 1.31 \\
\hline & Post & 10.50 & 1.10 & -.24 & .79 & 1.24 & 10.05 & 1.13 & -.09 & -.58 & .78 \\
\hline \multirow[t]{2}{*}{ Post-E1 } & Pre & 13.32 & 1.84 & -.97 & 1.29 & .68 & 13.67 & 2.10 & -1.12 & 1.15 & .88 \\
\hline & Post & 13.64 & 1.76 & -.36 & -.65 & .81 & 12.82 & 1.84 & -.61 & .24 & 1.04 \\
\hline \multirow[t]{2}{*}{ Post-E2 } & Pre & 14.00 & 1.75 & .01 & -1.24 & .69 & 14.36 & 1.59 & -.82 & .18 & 1.29 \\
\hline & Post & 14.23 & 1.60 & -.48 & -.12 & .80 & 13.14 & 1.93 & -.12 & -1.27 & .92 \\
\hline \multirow[t]{2}{*}{ Post-E3 } & Pre & 14.05 & 1.65 & -.15 & -1.01 & 1.03 & 14.45 & 1.68 & -.08 & -1.09 & .81 \\
\hline & Post & 14.55 & 1.63 & -.27 & -.98 & .84 & 13.18 & 1.82 & -.66 & 1.57 & .88 \\
\hline \multirow[t]{2}{*}{ 5' Post-E3 } & Pre & 9.23 & 1.11 & .88 & .44 & 1.23 & 9.55 & 1.18 & .07 & -1.52 & 1.04 \\
\hline & Post & 9.50 & 1.10 & .01 & -1.27 & .82 & 9.09 & 1.15 & .22 & -.87 & .90 \\
\hline
\end{tabular}

Cal= Calentamiento; $E=$ Ejercicio; $A=$ Asimetrí $; K=$ Kurtosis; $Z=$ Prueba de normalidad (Kolmogorov-Smirnov)

${ }^{\mathrm{a}} \mathrm{p}>.05$

Como se puede observar, los datos obtenidos para las medidas previas al calentamiento no mostraron una distribución normal, por lo que se precedió a utilizar técnicas estadísticas no paramétricas para evaluar las diferencias entre los grupos en cada momento experimental ( $U$ de Mann-Whitney) y entre las medidas pre y post para cada grupo (Wilcoxon). Los resultados indicaron que no existieron diferencias en las medidas pretest $(Z=-.59 ; p$ $>.05) \mathrm{ni}$ en las postest $(Z=-.47 ; p>.05)$. Por otro lado, tampoco hubo diferencias entre las medidas pretest y postest en los grupos control $(Z=-.45 ; p>.05)$ y experimental $(Z=-.58 ; p>.05)$.

Para analizar las restantes medidas se efectuaron análisis de la varianza (ANOVA) factorial. Los resultados indicaron que no hubo diferencias significativas en los efectos principales de la variables pre-post y grupo $(p>.05)$, aunque sí en los efectos de la interacción para las medidas posteriores al ejercicio $2\left(F_{[1,40]}=5.15 ; p<.05 ; \eta 2=.11 ; 1-\beta=.60\right)$ y el ejercicio $3\left(F_{[1,40]}=6.78 ; p<.05 ; \eta 2=.14 ; 1-\beta=.72\right)$. Además, hubo indicios de significación en los valores posteriores al ejercicio 1 $\left(F_{[1,40]}=3.95 ; p=.053 ; \eta 2=.09 ; 1-\beta=.49\right)$ y 5 minutos después del ejercicio $3\left(F_{[1,40]}=3.60 ; p=.065 ; \eta 2=.08 ; 1-\beta=.46\right)$. Además, la prueba de Levene indicó que no hubo problemas de homogeneidad de varianza cuyos valores oscilaron entre .01 y 1.53 , siendo no significativa en todos los casos $(p>.05)$. Por otro lado, las comparaciones simples (Bonferroni) mostraron diferencias significativas entre los grupos control y experimental (figura 1) en las medidas postest tras el ejercicio 2 y el ejercicio 3, e intragrupos entre el pretest y el postest en el caso del grupo experimental (figura 2) y tras los ejercicios 1,2 y 3.

Figura 1. Comparaciones simples para los valores de PSE previos y tras el programa actividad física entre los grupos control y experimental.
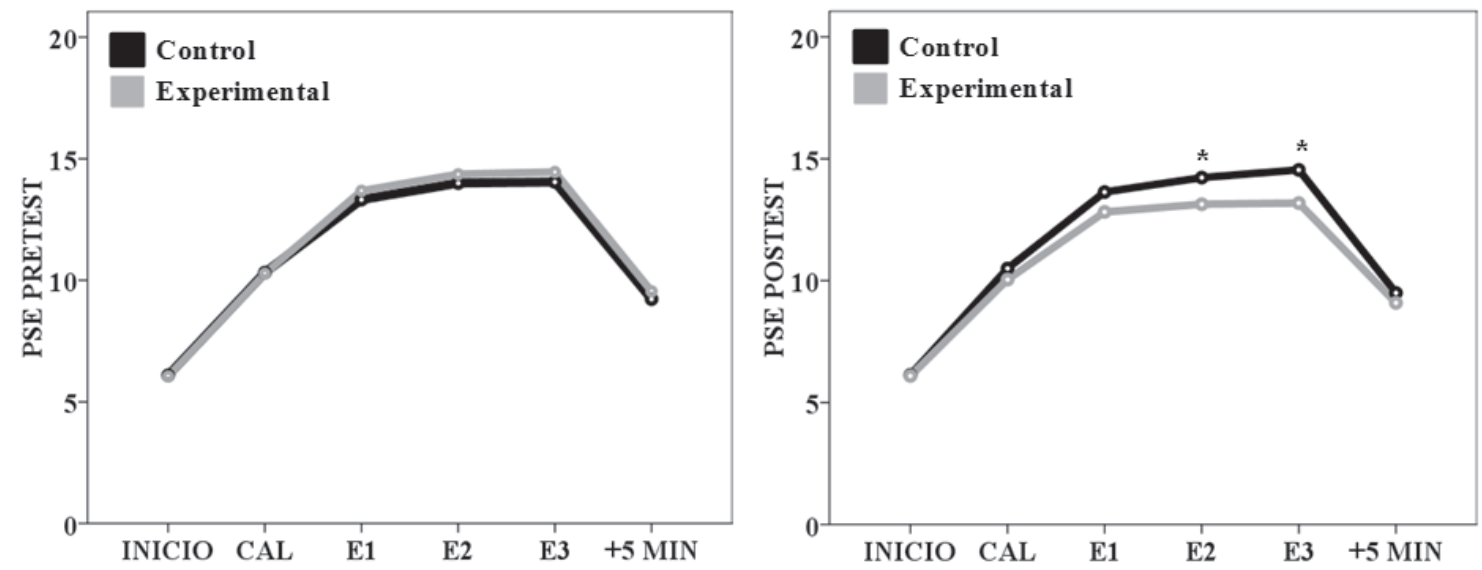
Figura 2. Comparaciones simples para los valores de PSE pretest y postest para el grupo experimental.

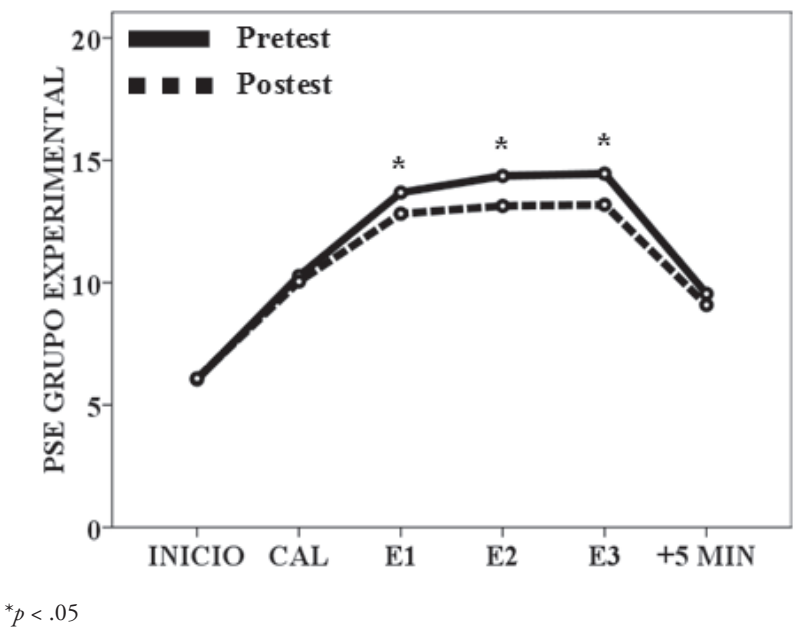

Índices de participación en el juego y de rendimiento en el juego (GPAI)

En la tabla 2 se muestran los estadísticos descriptivos y los valores de normalidad (Kolmogorov-Smirnov) para los índices de participación en el juego y rendimiento en el juego. Asimismo, la fiabilidad intraobservador (Kappa de Cohen), mostró valores superiores a .70 .

Tabla 2. Medias y desviaciones típicas para los valores IPJ e IRJ.

\begin{tabular}{|c|c|c|c|c|c|c|c|c|c|c|c|}
\hline & & \multicolumn{5}{|c|}{ Control } & \multicolumn{5}{|c|}{ Experimental } \\
\hline & & $M$ & $D T$ & $A$ & $K$ & $Z$ & $M$ & $D T$ & $A$ & $K$ & $Z$ \\
\hline \multirow[t]{2}{*}{ FS IPJ } & Pre & 6.33 & 4.23 & .78 & .86 & .91 & 4.71 & 2.34 & .56 & .37 & .87 \\
\hline & Post & 5.67 & 2.61 & .12 & -.26 & .74 & 6.67 & 2.95 & -.05 & -.10 & .77 \\
\hline \multirow[t]{2}{*}{ FS IRJ } & Pre & .23 & .15 & .47 & .49 & .67 & .25 & .22 & .84 & .70 & .76 \\
\hline & Post & .25 & .17 & .09 & -.91 & .45 & .49 & .17 & -.05 & -.15 & .98 \\
\hline \multirow[t]{2}{*}{ BM IPJ } & Pre & 6.57 & 3.51 & -.08 & .21 & .47 & 5.71 & 2.59 & .10 & -.35 & .56 \\
\hline & Post & 6.24 & 3.89 & .98 & .43 & .84 & 9.47 & 3.44 & .65 & -.27 & .65 \\
\hline \multirow[t]{2}{*}{ BM IRJ } & Pre & .33 & .20 & .41 & .35 & .80 & .41 & .18 & .12 & .29 & .99 \\
\hline & Post & .31 & .19 & .29 & -.28 & .44 & .62 & .24 & -.37 & -.86 & .73 \\
\hline \multirow[t]{2}{*}{ BC IPJ } & Pre & 7.95 & 4.27 & .87 & .89 & .62 & 7.48 & 3.39 & .11 & .94 & .67 \\
\hline & Post & 7.29 & 3.86 & .61 & -.20 & .71 & 10.04 & 3.94 & -.28 & -.73 & .54 \\
\hline \multirow[t]{2}{*}{ BC IRJ } & Pre & .31 & .16 & .23 & -.68 & .58 & .26 & .18 & .29 & -.85 & .63 \\
\hline & Post & .27 & .17 & .83 & .78 & .66 & .54 & .21 & .08 & .33 & .55 \\
\hline
\end{tabular}

$F S=$ Fútbol sala $; B M=$ Balonmano; $B C=$ Baloncesto IPJ= Índice de participación en el juego; $I R J=$ Índice de rendimiento en el juego

Como se puede observar en la tabla 2, los datos mostraron una distribución normal, por lo que se procedió a utilizar técnicas paramétricas para los análisis. Los resultados de los análisis de la varianza (ANOVA) factorial indican que no hubo diferencias significativas en el efecto principal grupo, aunque sí para la variable pre-post en IPJ para balonmano $\left(F_{[1,40]}=\right.$ $4.80 ; p<.05 ; \eta 2=.11 ; 1-\beta=.57)$, así como en IRJ para fútbolsala $\left(F_{[1,40]}=10.60 ; p<.01 ; \eta 2=.21 ; 1-\beta=.88\right)$, balonmano $\left(F_{[1,40]}=5.18 ; p<.05 ; \eta 2=.12 ; 1-\beta=.60\right)$ y baloncesto $\left(F_{[1,40]}\right.$ $=9.53 ; p<.01 ; \eta 2=.19 ; 1-\beta=.85)$. Asimismo, se apreciaron resultados significativos en los efectos de la interacción para las medidas IPJ para fútbol-sala $\left(F_{[1,40]}=6.67 ; p<.05 ; \eta 2=\right.$ $.14 ; 1-\beta=.71)$, balonmano $\left(F_{[1,40]}=6.85 ; p<.05 ; \eta 2=.15 ; 1-\right.$ $\beta=.72)$ y baloncesto $\left(F_{[1,40]}=4.18 ; p<.05 ; \eta 2=.10 ; 1-\beta=.51\right)$, así como para las medidas IRJ para fútbol-sala $\left(F_{[1,40]}=7.16\right.$; $p<.05 ; \eta 2=.15 ; 1-\beta=.74)$, balonmano $\left(F_{[1,40]}=7.33 ; p<.01\right.$; $\eta 2=.16 ; 1-\beta=.75)$ y baloncesto $\left(F_{[1,40]}=18.57 ; p<.001 ; \eta 2=\right.$ $.32 ; 1-\beta=.99)$. Además, la prueba de Levene indicó que no hubo problemas de homogeneidad de varianza cuyos valores oscilaron entre .01 y 2.02 , siendo no significativa en todos los casos $(p>.05)$.

Por otro lado, las comparaciones simples (Bonferroni) indicaron que no hubo diferencias significativas entre los grupos antes de la intervención. Sin embargo, tras ella, sí existieron en todos los factores salvo en IPJ para fútbol-sala. A su vez, se observaron diferencias significativas entre todas las medidas postest y pretest en el grupo experimental y en los tres deportes, aunque no en el grupo control (tabla 3). 
Tabla 3. Comparaciones entre e intra grupos para los valores IPJ e IRJ.

\begin{tabular}{|c|c|c|c|c|c|}
\hline & & \multicolumn{2}{|c|}{ Grupo } & \multicolumn{2}{|c|}{ Factor } \\
\hline & & $\begin{array}{c}\text { Control } \\
\text { Pre vs. Post }\end{array}$ & $\begin{array}{c}\text { Experimental } \\
\text { Pre vs. Post } \\
\end{array}$ & $\begin{array}{l}\text { Pretest } \\
\text { C vs. E } \\
\end{array}$ & $\begin{array}{l}\text { Postest } \\
\text { C vs. E }\end{array}$ \\
\hline \multirow[t]{2}{*}{ FS } & IPJ & .66 & $-1.52^{* *}$ & 1.62 & -1.00 \\
\hline & IRJ & -.02 & $-.24^{* * *}$ & -.02 & $-.24^{* * *}$ \\
\hline \multirow[t]{2}{*}{$\mathrm{BM}$} & IPJ & .33 & $-3.76^{* *}$ & .86 & $-3.24^{* *}$ \\
\hline & IRJ & .02 & $-.22^{* *}$ & -.08 & $-.31^{* * *}$ \\
\hline \multirow[t]{2}{*}{$\mathrm{BC}$} & IPJ & .66 & $-2.57^{*}$ & -.47 & $-2.76^{*}$ \\
\hline & IRJ & .05 & $-.28^{* * *}$ & .06 & $-.27^{* * *}$ \\
\hline \multicolumn{6}{|c|}{${ }^{*} p<.05 ;{ }^{* *} p<.01 ;{ }^{* * *} p<.001$} \\
\hline $\begin{array}{l}F S=F \\
\text { juego; }\end{array}$ & o-salc & $\begin{array}{l}M=\text { Balonman } \\
\text { de rendimient }\end{array}$ & $\begin{array}{l}C=\text { Baloncesto } ; \\
\text { el juego }\end{array}$ & de pa & ción en el \\
\hline
\end{tabular}

\section{Frecuencia cardiaca}

En las tabla 3 se muestran las medias y desviaciones típicas de la frecuencia cardiaca de trabajo antes y después de la intervención, así como el porcentaje de masa grasa y masa magra. Asimismo, se indican los valores de asimetría, curtosis y prueba de normalidad Kolmogorov-Smirnov.

Tabla 4. Medias y desviaciones típicas para los valores de FC. Medidas de dispersión y normalidad para los valores de FC

\begin{tabular}{|c|c|c|c|c|c|c|c|c|c|c|c|}
\hline & & \multicolumn{5}{|c|}{ Control } & \multicolumn{5}{|c|}{ Experimental } \\
\hline & & $M$ & $D T$ & $A$ & $K$ & $Z$ & $M$ & $D T$ & $A$ & $K$ & $Z$ \\
\hline \multirow[t]{2}{*}{$\mathrm{FC}$} & Pre & 174.54 & 9.49 & .07 & -.42 & .48 & 177.89 & 9.76 & -.88 & -.46 & 42 \\
\hline & Post & 173.62 & 8.87 & -.09 & .04 & .81 & 174.28 & 9.12 & 1.27 & 1.47 & .56 \\
\hline
\end{tabular}

${ }^{\mathrm{a}} p>.05$

$F C=$ Frecuencia cardiaca $;$ As = Asimetrí $; K=$ Kurtosis; $Z=$ Prueba de normalidad (Kolmogorov-Smirnov)

Los resultados de los análisis de la varianza (ANOVA) factorial indican que no hubo diferencias significativas en los efectos principales de la variable pre-post $\left(F_{[1,40]}=.94 ; p>.05\right.$; $\eta 2=.07 ; 1-\beta=.15)$ y gruро $\left(F_{[1,40]}=.23 ; p>.02 ; \eta 2=.07 ; 1-\beta=\right.$ $.60)$, ni tampoco para los efectos de la interacción $\left(F_{[1,40]}=.33\right.$; $p>.05 ; \eta 2=.03 ; 1-\beta=.08)$.

\section{Discusión}

El propósito del presente trabajo fue evaluar los efectos de un programa de actividad física basado en juegos colectivos reducidos (3 vs. 3) sobre la PSE en un grupo de chicas adolescentes. Asimismo, y como medidas de control, se observó la participación y la eficacia en la toma de decisiones durante el juego, así como la FC de trabajo. Los resultados encontrados han puesto de relieve una disminución significativa en la percepción del esfuerzo tras el periodo de intervención, lo que satisface el principal objetivo de la investigación. Estos resultados se encuentran en la línea de otras investigaciones que habían evaluado la incidencia de la experiencia de práctica en deportes colectivos, como Fuentes, Feu, Jiménez y CallejaGonzález (2013), que indicaron que la práctica acumulada en un deporte específico, en este caso en baloncesto-mini, generaba una menor percepción del esfuerzo percibido durante la competición en niños. Además, tienen relación con otros trabajos, como el de Dellal, Hill-Haas et al. (2011), que indicaron que la PSE era mayor en deportistas aficionados que en profesionales, sugiriendo que la condición de experto y el entrenamiento continuado tienen influencia en la percepción del esfuerzo con el que se valora la participación en una actividad.

La PSE ha sido una herramienta adecuada para evaluar los cambios en la valoración de la intensidad del ejercicio y la fatiga acumulada durante la sesión de trabajo, la cual ha sido ampliamente utilizada para analizar estos parámetros en diferentes formatos de juegos reducidos (Coutts et al., 2009; Hill-Hass et al., 2010; Rampinini et al., 2007). La disminución en el valor de esta variable estaría atendiendo a una menor percepción de intensidad de trabajo durante el ejercicio desarrollado. En esta línea, hay estudios que indicaron cambios en la percepción de esfuerzo tras efectuar modificaciones en la estructura de la actividad, estando asociados a cambios en la carga de trabajo soportada durante el juego (Casamichana et al., 2012; Köklü et al., 2013). Sin embargo, es difícil delimitar la naturaleza exacta de ciertas variables que pueden incidir en esos cambios. Es más fácil sugerir evaluar la incidencia de parámetros físicos de la carga de entrenamiento, sin embargo es más complicado analizar variables de tipo motivacional o el estrés cognitivo provocado por la toma de decisiones (Beniscelli y Torregrosa, 2010).

A pesar de esta complejidad, se considera que uno de los elementos que han podido influir en los resultados encontrados ha sido la disminución del estrés producido por las implicaciones cognitivas y la complejidad táctica a la que han sido 
sometidos los participantes. Tal y como consideran autores como Beniscelli y Torregrosa (2010), Guijarro et al. (2009) o Tenenbaum y Hutchinson (2007), la variabilidad en percepción de esfuerzo no se sitúa únicamente a nivel de condición física, sino también en el plano emocional o cognitivo. En esta investigación, el grupo experimental aumentó la participación en el juego y la eficacia de las acciones desarrolladas, lo que indica que existió una evolución positiva (adaptación) en aspectos relacionados con la toma de decisiones. Investigaciones anteriores habían puesto de manifiesto que metodologías que el entrenamiento con juegos reducidos sería adecuado para potenciar el aprendizaje de las acciones técnico-tácticas en los deportes colectivos y mejorar la eficacia en la toma de decisiones (Balakishnan et al., 2011; Chatzopoulos, Drakou, Kotzamanidou y Tsorbatzoudis, 2006; Harvey et al., 2010; Mesquita et al., 2012). Esto podría ser coherente con la hipótesis que indicaría que los participantes habrián tenido una adecuada adaptación a las exigencias del juego y habrían disminuido el estrés generado por las exigencias de las situaciones del juego a las que habría que enfrentarse.

El formato de juego reducido empleado fue 3 vs. 3, seńalado en diversos trabajos como un buen modelo para aumentar el número de acciones por jugador y adecuado para incrementar la carga de decisiones durante el juego (Da Silva et al., 2011; Duarte et al., 2009; Frencken et al., 2013; Jones y Drust, 2007; Vaeyens, Lenoir, Williams, Mazyn y Philippaerts, 2007). De hecho, en un estudio efectuado por Katis y Kellis (2009) se puso de manifiesto que situaciones de 3 vs. 3 eran más eficaces para la mejora de las acciones técnico-tácticas que otras como el 6 vs. 6. Por ello, este formato de juego reducido podría haber sido eficaz para el aprendizaje de los deportes colectivos trabajados, mejorando los procesos de toma de decisión y acelerando la asimilación de las demandas cognitivas del juego (Casamichana y Castellano, 2010; HillHaas, Dawson et al., 2011). Asimismo, en diversos estudios se ha puesto de relieve que cuando en los juegos reducidos se incrementa el número de decisiones aumenta la percepción del esfuerzo realizado (Dellal, Chamari et al., 2011; Dellal et al., 2012; Hill-Hass et al., 2011). Sin embargo, los datos obtenidos en este trabajo indican que, tras la intervención, los valores de los índices de participación y eficacia en el juego fueron más altos. Esto contribuiría a considerar que el esfuer- zo percibido para tomar las decisiones y hacerlo con un mayor éxito ha disminuido en el grupo experimental.

Asimismo, la FC media con la que se ha trabajado durante los ejercicios antes y después de la intervención fue similar. Sin embargo, no se han extraído otros datos que pudieran confirmar si se produjeron adaptaciones orgánicas al ejercicio, como el consumo máximo de oxígeno. No obstante, la literatura existente indica que las adaptaciones cardiovasculares hubieran generado una disminución de la frecuencia cardiaca en reposo (Cordova, Villa, Sureda, Rodriguez-Marroyo y Sánchez-Collado, 2012), lo que hubiera implicado un aumento de la frecuencia cardiaca de reserva. En caso de haberse producido tales adaptaciones, la frecuencia cardiaca en la evaluación final indicaría que se habría trabajado un ritmo mayor. Por tanto, aunque es una limitación del estudio, podemos considerar un indicador de que se ha trabajado a una intensidad similar, o en todo caso nunca menor, en la evaluación efectuado tras la intervención.

Por lo tanto, como limitación del estudio hay que señalar que no se han controlado de forma exhaustiva variables de condición física (Polman, Bloomfield y Edwards, 2009) que podrían aportar más información sobre los resultados del trabajo, aunque se considera que se han obtenido hallazgos en la línea de otros autores que habían puesto de manifiesto la naturaleza multidimensional de los factores condicionan la percepción del esfuerzo durante un ejercicio físico. Sin embargo, sí ha pretendido aportar datos sobre la cuestión objeto de estudio diferenciando la intensidad física del trabajo realizado de las demandas cognitivas soportadas durante el juego, lo cual es interesante. En futuros trabajos, se sugiere seguir profundizando en los factores responsables de la variabilidad en percepción de esfuerzo, dada la importancia que tienen estos procesos en el control del trabajo en el ámbito deportivo.

Por tanto, el presente estudio pone de manifiesto, en un grupo de chicas adolescentes no entrenadas, que la práctica física continuada a través de juegos reducidos ha generado una adecuada adaptación a las demandas del juego colectivo, y ha disminuido la percepción del esfuerzo soportado durante la práctica de estos ejercicios físico. Este trabajo sugiere que los procesos de toma de decisión en este tipo de deportes tienen un impacto parcial sobre la fatiga percibida a lo largo de la actividad y puede ser mejorada a través del entrenamiento.

\section{Referencias}

1. Abrantes, C.I., Nunes, M.I., Maças, V.M., Leite, N.M. y Sampaio, J.E. (2012). Effects of the number of players and game type constraints on heart rate, rating of perceived exertion and technical actions of smallsided soccer games. Journal of Strength and Conditioning Research, 26(4), 976-981.

2. Alexiou, H. y Coutts, A. (2008). A comparison of methods used for quantifying internal training load in women soccer players. International Journal of Sports Physiology and Performance, 3, 320330 .
3. Araújo, D. (2013). The study of decision-making behavior in sport. $R I$ CYDE. Revista Internacional de Ciencias del Deporte, 9(31), 1-4.

4. Balakishnan, M., Rengasamy, S. y Aman, M. S. (2011). Effect of Teaching Games for Understanding approach on students' cognitive learning outcome. World Academy of Science, Engineering and Technology, 53, 961-963

5. Beniscelli, V. y Torregrosa, M. (2010). Componentes del esfuerzo percibido en fútbol de iniciación. Cuadernos de psicología del deporte, 10(1), $7-22$. 
6. Borg, G. (1970) Perceived exertion as an indicator of somatic stress. Scandinavian Journal of Rehabilitation Medicine, 2(2), 92-98.

7. Borg, G.A. (1973). Perceived exertion: a note on "history" and methods. Medicine and Science in Sports, 5, 90-93.

8. Borg, G.A. (1982). Psychological bases of perceived exertion. Medicine and Science in Sports and Exercise, 14(5), 377-381.

9. Casamichana, D. y Castellano, J. (2009). Análisis de los diferentes espacios individuales de interacción y los efectos en las conductas motrices de los jugadores. Aplicaciones al entrenamiento en fútbol. Motricidad. European Journal of Human Movement, 15(23), 143-167.

10. Casamichana, D. y Castellano, J. (2010). Time-motion, heart rate, perceptual and motor behaviour demands in small-sides soccer games: Effects of pitch size. Journal of Sports Sciences, 28(14), 1615-1623.

11. Casamichana, D., Castellano, J., Blanco-Villaseñor, Á. y Usabiaga, O. (2012). Estudio de la Percepción Subjetiva del Esfuerzo en Tareas de Entrenamiento en Fútbol a través de la Teoría de la Generalizabilidad. Revista de Psicología del Deporte, 21(1), 35-40.

12. Casamichana, D., Castellano, J. y Dellal, A. (2013). Influence of different training regimes on physical and physiological demands during small-sided soccer games: continuous vs. intermittent format. The Journal of Strength \& Conditioning Research, 27(3), 690-697.

13. Casamichana, D., San Román-Quintana, J., Calleja-González, J. y Castellano, J. (2013). Utilización de la limitación de contactos en el entrenamiento en fútbol:¿ afecta a las demandas físicas y fisiológicas?. RICYDE. Revista Internacional de Ciencias del Deporte, 9(33), 208-221.

14. Chatzopoulos, D., Drakou, A., Kotzamanidou, M., \& Tsorbatzoudis, H. (2006). Girls' soccer performance and motivation: games Vs Technique approach. Perceptual \& Motor Skills, 103(2), 463-470.

15. Cordova, A., Villa, G., Sureda, A., Rodriguez-Marroyo, J.A. y Sánchez-Collado, M.P. (2012). Actividad física y factores de riesgo cardiovascular de niños españoles de 11-13 años. Revista Española de Cardiología, 65(7), 620-626.

16. Corrêa, U.C., Silva, A.S. y Paroli, R. (2004). Efeitos de diferentes métodos de ensino na aprendizagem do futebol de saláo. Revista Motriz, Rio Claro, 10(2), 79-88.

17. Coutts, A.J., Rampinini, E., Marcora, S.M., Castagna, C., \& Impellizzeri, F.M. (2009). Heart rate and blood lactate correlates of perceived exertion during small-sided soccer games. The Journal of Sports Science and Medicine, 12(1), 79-84.

18. Cuadrado-Reyes, J., Chirosa-Ríos, L.J., Chirosa-Ríos, I., Martin-Tamayo, I. y Aguilar-Martínez, D. (2012). La percepción subjetiva del esfuerzo para el control de la carga de entrenamiento en una temporada en un equipo de balonmano. Revista de Psicología del Deporte, 21(2), 331-339.

19. Da Silva, C., Impellizzeri, F., Natali, A., De Lima, J., Bara-Filho, M., Silami-García, E. y Marins, J. (2011). Exercise intensity and technical demands of small-sided games in young brazilian soccer players: effect of number of players, maturation, and reliability. Journal of Strength and Conditioning Research, 25(10), 2746-2751.

20. Dellal, A., Chamari, K., Owen, A.L., Wong, D.P., Lago-Penas, C. y Hill-Haas, S. (2011). Influence of technical instructions on the physiological and physical demands of small-sided soccer games. European Journal of Sport Science, 11(5), 341-346.

21. Dellal, A., Chamari, K., Pintus, A., Girard, O., Cotte, T. y Keller, D. (2008). Heart rate responses during small-sided games and short intermittent running training in elite soccer players: A comparative study. Journal of Strength and Conditioning Research, 22(5), 1449-1457.

22. Dellal, A., Drust, B. y Lago-Penas, C. (2012). Variation of activity demands in small-sided soccer games. International Journal of Sport Medicine, 33(5), 370-375.

23. Dellal, A., Hill-Haas, S., Lago-Penas, C. y Chamari, K. (2011). Smallsided games in soccer: amateur vs. professional players' physiological responses, physical, and technical activities. The Journal of Strength \& Conditioning Research, 25(9), 2371-2381.
24. Dellal, A., Lago-Penas, C., Wong, D.P. y Chamari, K. (2011). Effect of the number of the ball contacts within bouts of 4 vs. 4 small-sided games. International Journal of Sport Physiology and Performance, 6(3), 322-333.

25. Dellal, A., Owen, A., Wong, D.P., Krustrup, P., Van Exsel, M. y Mallo, J. (2012). Technical and physical demands of small vs. large sided games in relation to playing position in elite soccer. Human Movement Science, 31(4), 957-969.

26. Duarte, R., Batalha, N., Folgado, H. y Sampaio, J. (2009). Effects of exercise duration and number of players in heart rate responses and technical skills during futsal small-sided games. The Open Sports Sciences Journal, 2, 1-5.

27. Eurofit (1993). Eurofit Tests of Physical Fitness (2a ed.). Strasbourg: Committee of Experts on Sports Research.

28. Faulkner, J., Parfitt, G. y Eston, R. (2008). The rating of perceived exertion during competitive running scales with time. Psychophysiology, 45, 977-985.

29. Feriche, B., Chicharro, J. L., Vaquero, A. F., Perez, M. y Lucia, A (1998). The use of a fixed value of RPE during a ramp protocol. Comparison with the ventilatory threshold. The Journal of Sports Medicine and Physical Fitness, 38(1), 35-38.

30. Frencken, W., Van Der Plaats, J., Visscher, C. y Lemmink, K. (2013). Size matters: pitch dimensions constrain interactive team behaviour in soccer. Journal of Systems Science and Complexity, 26(1), 85-93.

31. Fuentes, M., Feu, S., Jiménez, C. y Calleja-González, J. (2013). Perceived exertion effort in mini basketball players and its relationship with training volume. Revista de Psicología del Deporte, 22(1), 205-208.

32. George, J.D., Fisher, A.G. y Vehrs, P.R. (2007). Tests y pruebas físicas (4a ed.). Barcelona: Paidotribo.

33. Guijarro, E., de la Vega, R., y del Valle, S. (2009). Ciclo menstrual, rendimiento y percepción del esfuerzo en jugadoras de fútbol de élite. Revista Internacional de Medicina y Ciencias de la Actividad Física y el Deporte, 9(34), 96-104.

34. Harvey, S., Cushion, C.J., Wegis, H.M. y Massa-Gonzalez, A.N. (2010). Teaching games for understanding in American high-school soccer: a quantitative data analysis using the Game Performance Assessment Instrument. Physical Education \& Sport Pedagogy, 15(1), 2954.

35. Hill-Haas, S.V., Coutts, A.J., Dawson, B.T. y Rowsell, G.J. (2010). Time-motion characteristics and physiological responses of small-sided games in elite youth players: the influence of player number and rule changes. Journal of Strength and Conditioning Research, 24(8), 2149 2156.

36. Hill-Haas, S.V, Dawson, B.T., Impellizzeri, F.M. y Coutts, A. J. (2011). Physiology of Small-Sided Games Training in Football A Systematic Review. Sports Medicine, 41(3), 199-220.

37. Hill-Hass, S.V., Rowsell, G.J., Dawson, B.T. y Coutts, A.J. (2009). Acute physiological responses and time-motion characteristics of two small-sided training regimes in youth soccer players. Journal of Strength and Conditioning Research, 23(1), 111-115.

38. Impellizzeri, F. M., Rampinini, E., Coutts, A. J., Sassi, A., y Marcora, S. M. (2004). Use of RPE-based training load in soccer. Medicine and Science in Sports and Exercise, 36(6), 1042-1047.

39. Jones, S. y Drust, B. (2007). Physiological and technical demands of 4 v 4 and 8 v 8 games in elite youth soccer players. Kinesiology, 39(2), $150-156$.

40. Katis, A. y Kellis, E. (2009). Effects of small-sided games on physical conditioning and performance in young soccer players. Journal of Sports Science and Medicine, 8, 374-380.

41. Kilpatrick, M.W., Bortzfield, A.L. y Giblin, L. M. (2012). Impact of aerobic exercise trials with varied intensity patterns on perceptions of effort: An evaluation of predicted, in-task, and session exertion. Journal of Sports sciences, 30(8), 825-832. 
42. Köklü, Y., Albayrak, M., Keysan, H., Alemdaroğlu, U. y Dellal, A. (2013). Improvement of the physical conditioning of young soccer players by playing small-sided games on different pitch size-special reference to physiological responses. Kinesiology, 45(1), 41-47.

43. Léger, L.A., Mercier, D., Gadoury, C. y Lambert, J. (1988). The multistage 20 metre shuttle run test for aerobic fitness. Journal of Sports Sciences, 6, 93-101.

44. Mesquita, I., Farias, C. y Hastie, P. (2012). The impact of a hybrid Sport Education-Invasion Games Competence Model soccer unit on students' decision making, skill execution and overall game performance. European Physical Education Review, 18(2), 205-219.

45. Milanez, V.F., Lima, S., Gobatto, C.A., Perandini, L.A., Nakamura, F.Y. y Ribeiro, L.F.P. (2011). Correlates of session-rate of perceived exertion (RPE) in a karate training session. Science \& Sports, 26(1), 38 43.

46. Mitchell, S.A., Oslin, J.L. y Griffin, L.L. (1995). The effects of two instructional approaches on game performance. Pedagogy in Practice: Teaching \& Coaching in Physical Education \& Sports, 1(1), 36-48.

47. Monteiro, W.D., Farinatti, P.T., de Oliveira, C.G. y Araújo, C.G.S (2011). Variability of cardio-respiratory, electromyographic, and perceived exertion responses at the walk-run transition in a sample of young men controlled for anthropometric and fitness characteristics. European journal of applied physiology, 111(6), 1017-1026.

48. Oslin, J.1., Mitchell, S.A. y Griffin, L. 1. (1998). The Game Performance Assessment Instrument (GPAI): development and preliminary validation. Journal of Teaching in Physical Education, 17(2), 231-243.

49. Polman, R., Bloomfield, J. y Edwards, A. (2009). Effects of SAQ training and small-sided games on neuromuscular functioning in untrained subjects. International Journal of Sports Physiology and Performance, 4(4), 494-505.

50. Rampinini, E., Impellizzeri, F.M., Castagna, C., Abt, G., Chamari, K., Sassi, A. y Marcora, S.M. (2007). Factors influencing physiological responses to small-sided soccer games. Journal of Sports Sciences, 25(6), 659-666.

51. Sampaio, J., Abrantes, C., Leite, N. (2009). Power, heart rate and perceibed exertion responses to $3 \times 3$ and $4 \times 4$ basketball small sided games. Revista de Psicología del Deporte, 18(3), 463-467.

52. Skatrud-Mickelson, M., Benson, J., Hannon, J.C. y Askew, E.W. (2011). A comparison of subjective and objective measures of physical exertion. Journal of Sports Sciences, 29(15), 1635-1644

53. Tenenbaum, G., Basevitch, I., Gershgoren, L. y Filho, E. (2013). Emotions-decision-making in sport: Theoretical conceptualization and experimental evidence. International Journal of Sport and Exercise Psychology, 11(2), 151-168.

54. Tenenbaum, G. y Hutchinson, J. C. (2007). A social cognitive perspec tive of perceived sustained sffort. En G. Tenenbaum y R. C. Eklund (Eds.) Handbook of sport psychology, (pp. 560-577). Nueva York: Wiley.

55. Toh, S., Guelfi, K., Wong, P. y Fournier, P. (2011). Energy expenditure and enjoyment of small-sided soccer games in overweight boys. $\mathrm{Hu}$ man Movement Science, 30(3), 636-647.

56. Vaeyens, R., Lenoir, M., Williams, A.M., Mazyn, L. y Philippaerts, R.M. (2007). The effects of task constraints on visual search behavior and decision-making skill in youth soccer players. Journal of Sport and Exercise Psychology, 29(2), 147-169.

57. Vila-Maldonado, S., García, L.M. y Contreras, O.R. (2012). La investigación del comportamiento visual, desde el enfoque perceptivocognitivo y la toma de decisiones en el deporte. Journal of Sport and Health Research, 4(2), 137-156.

58. Vilar, L., Araújo, D., Davids, K. y Button, C. (2012). The role of ecological dynamics in analysing performance in team sports. Sports $\mathrm{Me}$ dicine, 42(1), 1-10

59. Wright, S., McNeill, M., Fry, J. y Wang, J. (2005). Teaching teachers to play and teach games. Physical Education \& Sport Pedagogy, 10(1), 61-82. 